\title{
Physiological Effect of Prone Positioning in Mechanically Ventilated SARS- CoV-2 Infected Patients with Severe ARDS: Preliminary Analysis of an Observational Study
} Avishek Roy*1, Srikant Behera*1, Aparna Pande ${ }^{1}$, Anirban Bhattacharjee ${ }^{1}$, Amrita Bhattacharyya $^{1}$, Dalim $\mathrm{K}$ Baidya ${ }^{1}$, Rahul $\mathrm{K}$ Anand ${ }^{1}$, Bikash $\mathrm{R}$ Ray $^{1}$, Rajeshwari Subramaniam $^{1}$, Souvik Maitra ${ }^{1}$

1 Department of Anaesthesiology, Pain Medicine \& Critical Care, All India Institute of Medical Sciences, New Delhi

* Both can be considered as 'first author' as they contributed equally to project.

Correspondence to:

Dr. Souvik Maitra MD, DNB, EDIC

Assistant Professor,

Department of Anaesthesiology, Pain Medicine \& Critical Care,

Room No: 5013, Teaching Block

All India Institute of Medical Sciences, Ansari Nagar,

New Delhi- 110029

Telephone: +91-11- 26593212

Email: souvikmaitra@aiims.edu

Key words: SARS- CoV-2, ARDS, COVID-19, prone position, respiratory mechanics

Source of Support: None

Conflict of interests: Nil 
Abstract

Prone position ventilation has been shown to decrease mortality and improve oxygenation in ARDS patients. With best of our knowledge, no study reported physiological effect of prone position in SARS- CoV-2 infected ARDS patients. In this prospective observational study, data of $n=20$ consecutive laboratory confirmed SARS- CoV-2 patients with severe ARDS as per Berlin definition was included. Data of 20 patients analyzed with a median (Interquartile range, IQR) age of 56 (45.5- 67) y and median (IQR) P/F ratio of 56 (54-66) with a median (IQR) PEEP of 12 (12- 14) before initiation of prone position. Seventy-five percentage (95\% CI 53.1- 88.8) patients were prone responders at $16 \mathrm{~h}$ prone session and 50 (95\% CI 29.970.1) $\%$ patients were sustained responders. There was a significant decrease in plateau airway pressure $(\mathrm{p}<0.0001)$, peak airway pressure $(\mathrm{p}<0.0001)$ and driving pressure $(\mathrm{p}<0.0001)$ and increase in static compliance $(\mathrm{p}=0.001), \mathrm{P} / \mathrm{F}$ ratio $(\mathrm{p}<0.0001), \mathrm{PaO}_{2}$ $(\mathrm{p}=0.0002)$ and $\mathrm{SpO}_{2}(\mathrm{p}=0.0004)$ at $4 \mathrm{~h}$ and $16 \mathrm{~h}$ since initiation of prone session and also after return of supine position. Prone position in SARS- CoV-2 infected severe ARDS patients is associated with improvement in lung compliance and oxygenation in two- third of the patients and persisted in half of the patients. 
Introduction

Since the diagnosis of first case in December 2019, SARS- CoV-2 has infected more that 16 million and caused fatality in more than 600,000 people worldwide. Early data from China reported that around 5\% of all laboratory confirmed cases become critically ill. [1]. Although the overall case fatality rate (CFR) ranges between 2.3 to $7.4 \%$, mortality in critically ill can reach $26 \%$ [2].

Early reports from Italy indicated that among the different phenotypes of ARDS in SARSCoV-2 pneumonia that have been proposed, the ' $L$ ' phenotype has normal lung compliance and lung weight but leads to hypoxemia due to the loss of hypoxic pulmonary vasoconstriction, later it progresses to ' $\mathrm{H}$ ' phenotype with low compliance and increased lung weight [3].

Prone position ventilation has been shown to decrease mortality and improve oxygenation in ARDS patients. In mechanically ventilated patients of severe ARDS with $\mathrm{PaO}_{2} / \mathrm{FiO}_{2}(\mathrm{P} / \mathrm{F})$ ratio $<150$, prone position for at least 4 days decreased 28-day mortality by almost $50 \%$ [4]. Prone position reduces lung strain and stress, leads to more homogenized distribution of lung aeration and recruitment of dorsal alveoli, thus, leading to improvement in oxygenation [5]. With best of our knowledge, no study reported physiological effect of prone position in SARS-CoV-2 infected ARDS patients. Hence, in this preliminary analysis of an observational study, physiological effect of prone position in SARS- CoV-2 infected severe ARDS patients have been reported.

Methods

Permission from the Institute Ethics Committee was obtained before recruitment of first patient and consent was obtained from legally acceptable representative of all recruited patients. In this prospective observational study, data of $n=20$ consecutive laboratory confirmed SARS- CoV-2 patients with severe ARDS as per Berlin definition was included. 
As per ICU protocol, in the absence of contraindication, all mechanically ventilated ARDS patients with $\mathrm{PaO}_{2} / \mathrm{FiO}_{2}<150$ were placed in at least 16h/day prone position for consecutive days till the criteria is met. Demographic characteristics, baseline respiratory mechanics and blood gas data were collected before initiation of prone position, after $4 \mathrm{~h}$ and $16 \mathrm{~h}$ of prone position and after $4 \mathrm{~h}$ of return of supine position. Positive end expiratory pressure (PEEP) was titrated as per ARDSNet protocol PEEP- $\mathrm{FiO}_{2}$ table. Prone responders were defined by $20 \%$ increase in $\mathrm{PaO}_{2} / \mathrm{FiO}_{2}$ ratio during the prone session and sustained responders were defined by $20 \%$ increase in $\mathrm{PaO}_{2} / \mathrm{FiO}_{2}$ ratio $4 \mathrm{~h}$ after return of supine position.

All collected data were entered in a Microsoft Excel datasheet. Categorical data were presented as as absolute numbers or percentages and non- parametric data were presented as median and IQR (inter-quartile range). Unrelated data (between prone responders and nonresponders) were compared by Mann- Whitney U test or Chi-square test as applicable. Longitudinal variables were compared by Friedman's test and multiple comparisons were performed by Dunn's test. A two-sided p value $<0.05$ was considered as significant. All statistical analyses were performed using GraphPad Prism version 8.0.0 for Mac OS, (GraphPad Software, San Diego, California USA).

Results

In this observational study, data of 20 patients analyzed with a median (IQR) age of 56 (45.567) y and median (IQR) predicted body weight of $60(55-62.5) \mathrm{kg}$. Baseline respiratory mechanics data is presented in table 1. All included patients had severe ARDS with median (IQR) P/F ratio of 56 (54- 66) with a median (IQR) PEEP of 12 (12- 14) before initiation of prone position. Median (IQR) SOFA score was 7.5 (5.5-9) at the time of inclusion. Seventyfive percentage (95\% CI 53.1- 88.8) patients were prone responders at $16 \mathrm{~h}$ prone session and 50 (95\% CI 29.9- 70.1) \% patients were sustained prone responders after return to supine position. Prone responders had significantly higher baseline respiratory compliance $(\mathrm{p}=0.03$, 
Mann Whitney U test) but all other respiratory and blood gas variables were similar between responders and non- responders.

There was a significant decrease in plateau airway pressure $(\mathrm{p}<0.0001)$, peak airway pressure $(\mathrm{p}<0.0001)$ and driving pressure $(\mathrm{p}<0.0001)$ and increase in static compliance $(\mathrm{p}=0.001)$ at $4 \mathrm{~h}$ and $16 \mathrm{~h}$ since initiation of prone session and also after return of supine position. Change in respiratory mechanics parameters from baseline are reported in figure $1 . \mathrm{P} / \mathrm{F}$ ratio $(\mathrm{p}<0.0001)$, $\mathrm{PaO}_{2}(\mathrm{p}=0.0002)$ and $\mathrm{SpO}_{2}(\mathrm{p}=0.0004)$ increased from baseline and persisted in supine position also (figure 1). Noradrenalin requirement didn't change during the prone session $(\mathrm{p}=0.20)$. Percentages of changes in static compliance significantly correlated with $\mathrm{P} / \mathrm{F}$ ratio after return of supine position $\left(\mathrm{r}^{2}=0.62, \mathrm{p}=0.0034\right)$ but not at $4 \mathrm{~h}(\mathrm{p}=0.14)$ and $16 \mathrm{~h}(\mathrm{p}=0.20)$. Percentages of changes in $\mathrm{P} / \mathrm{F}$ ratio and driving pressure at $16 \mathrm{~h}\left(\mathrm{r}^{2}=-0.47, \mathrm{p}=0.04\right)$ and after return of supine position $\left(\mathrm{r}^{2}=-0.59, \mathrm{p}=0.0089\right)$ were significantly correlated; but no correlation was found at $4 \mathrm{~h}(\mathrm{p}=0.09)$.

Baseline static compliance was a predictor of prone response with reasonable accuracy [AUROC (95\% CI) $0.82(0.59-1.00)]$. Static compliance $<14$ predicted no response from prone position with sensitivity $(95 \% \mathrm{CI})$ and specificity $(95 \% \mathrm{CI})$ of $80(37.6-99) \%$ and 73.3 (48.1- 89.1) \% respectively. At the time of writing of this manuscript, 4 patients [proportion (95\% CI) $22.2(9-45.2) \%$ ] were discharged from hospital and 2 patients were still in the hospital.

Discussion

We have found that around $75 \%$ of the SARS- CoV-2 infected patients with severe ARDS responded with $16 \mathrm{~h}$ prone position in terms of oxygenation. Overall there is improvement in lung mechanics in terms of static compliance, driving pressure and plateau pressure without any changes in the hemodynamic support. In our series, all the included patients had 'stiff lung' as evident by low static compliance. Previous studies reported a variable change in 
respiratory system compliance in prone position in ARDS patients [6], whereas we have found a significant decrease in driving pressure and static compliance. Recruitment of the dorsal lung region was the biologically plausible mechanism of improvement in static compliance as both driving pressure and compliance were correlated with change in $\mathrm{P} / \mathrm{F}$ ratio [7]. We have found that these correlations were present even after return of supine position which indicated a sustained lung recruitment in SARS- CoV-2 infected patients. Determination of baseline static compliance is important as it was a predictor of absence of response from prone position and these patients may be subjected to extra-corporeal membrane oxygenation early in the course of disease. Our study has few limitations such as sample size was small, and we couldn't assess the effect of prone position on chest wall and lung compliance separately as esophageal manometry was not used.

Conclusion

Prone position in SARS- CoV-2 infected severe ARDS patients is associated with improvement in lung compliance and oxygenation in two- third of the patients and persisted in half of the patients. 
Table 1: Respiratory Mechanics data at the time of enrolment (before prone position)

\begin{tabular}{|c|c|c|c|c|}
\hline & $\begin{array}{l}\text { All Patients } \\
(\mathrm{n}=20)\end{array}$ & $\begin{array}{l}\text { Responders } \\
(n=15)\end{array}$ & $\begin{array}{l}\text { Non- } \\
\text { responders } \\
(n=5)\end{array}$ & Significance \\
\hline \multicolumn{5}{|c|}{ Demographic Characteristics } \\
\hline Age (y) & $56(45.5-67)$ & $51(43-65)$ & $65(50-74)$ & $\mathrm{p}=0.24$ \\
\hline $\begin{array}{l}\text { Predicted Body } \\
\text { Weight }(\mathrm{kg})\end{array}$ & $60(55-62.5)$ & $60(55-64)$ & $60(54-62.5)$ & $\mathrm{p}=0.69$ \\
\hline Sex (Male/ Female) & $15 / 5$ & $11 / 4$ & $4 / 1$ & $p>0.99$ \\
\hline \multicolumn{5}{|l|}{ Respiratory Mechanics } \\
\hline Tidal Volume & $350(340-360)$ & $350(350-360)$ & $340(320-390)$ & $\mathrm{p}=0.85$ \\
\hline Peak Airway Pressure & $50(46-53)$ & $48(44-52)$ & $50(49-55)$ & $\mathrm{p}=0.27$ \\
\hline $\begin{array}{l}\text { Plateau Airway } \\
\text { Pressure }\end{array}$ & $36(35-37)$ & $36(35-38)$ & $36(31-38)$ & $\mathrm{p}=0.72$ \\
\hline $\begin{array}{l}\text { Positive End- } \\
\text { expiratory Pressure }\end{array}$ & $12(12-14)$ & $14(12-15)$ & $12(11-13)$ & $\mathrm{p}=0.17$ \\
\hline Respiratory Rate & $28(24-30)$ & $25(24-28)$ & $30(24-30)$ & $\mathrm{p}=0.24$ \\
\hline Static Compliance & $16(11-20.5)$ & $18(10-21)$ & $10(8-16)$ & $\mathrm{p}=0.03$ \\
\hline Driving Pressure & $23(20-25.5)$ & $21(20-26)$ & $24(17-25)$ & $\mathrm{p}=0.65$ \\
\hline \multicolumn{5}{|l|}{ Arterial Blood Gas } \\
\hline $\mathrm{SpO}_{2}$ & $88(85-92)$ & $90(86-92)$ & $84(84-91)$ & $\mathrm{p}=0.25$ \\
\hline $\mathrm{PaO}_{2}$ & $56(54-63)$ & $56(54-64)$ & $55(51-73)$ & $\mathrm{p}=0.54$ \\
\hline P/ F Ratio & $56(54-66)$ & $56(54-65)$ & $56(51-80)$ & $\mathrm{p}=0.88$ \\
\hline
\end{tabular}




\begin{tabular}{|l|l|l|l|l|l|}
\hline $\mathrm{pH}$ & $7.30 \quad(7.27-$ & $7.29 \quad(7.24-$ & $7.30 \quad(7.28-$ & $\mathrm{p}=0.69$ \\
& $7.34)$ & $7.34)$ & $7.37)$ & \\
\hline $\mathrm{PaCO}_{2}$ & $44(38-49)$ & $44(38-57)$ & $46(35-49)$ & $\mathrm{p}=0.72$ \\
\hline $\begin{array}{l}\text { Noradrenalin Infusion } \\
(\mathrm{mcg} / \mathrm{m})\end{array}$ & $0(0-5)$ & $5(0-10)$ & $5(0-8.75)$ & $\mathrm{p}=0.84$ \\
\hline
\end{tabular}

Figure Legends

Figure 1: Before- after line graph showing respiratory mechanics and blood gas parameters in all patients [p values for Dunn's multiple comparison (baseline vs other time points) after Friedman's test reported]

References

1. Wu Z, McGoogan JM. Characteristics of and important lessons from the coronavirus disease 2019 (COVID-19) outbreak in China: summary of a report of 72314 cases from the Chinese Center for Disease Control and Prevention. Jama. 2020 Apr 7;323:1239-42.

2. Yang X, Yu Y, Xu J, Shu H, Liu H, Wu Y, Zhang L, Yu Z, Fang M, Yu T, Wang Y. Clinical course and outcomes of critically ill patients with SARS-CoV-2 pneumonia in Wuhan, China: a single-centered, retrospective, observational study. The Lancet Respiratory Medicine. 2020 Feb 24.

3. Grasselli G, Zangrillo A, Zanella A, Antonelli M, Cabrini L, Castelli A, Cereda D, Coluccello A, Foti G, Fumagalli R, Iotti G. Baseline characteristics and outcomes of 1591 patients infected with SARS-CoV-2 admitted to ICUs of the Lombardy Region, Italy. JAMA. 2020;323:1574-81.

4. Guérin C, Reignier J, Richard JC, Beuret P, Gacouin A, Boulain T, Mercier E, Badet M, Mercat A, Baudin O, Clavel M. Prone positioning in severe acute respiratory distress syndrome. New England Journal of Medicine. 2013;368:2159-68.

5. Mentzelopoulos Mentzelopoulos SD, Roussos C, Zakynthinos SG. Prone position reduces lung stress and strain in severe acute respiratory distress syndrome. European Respiratory Journal. 2005;25:534-44. 
medRxiv preprint doi: https://doi.org/10.1101/2020.09.16.20195958; this version posted September 18, 2020. The copyright holder for this preprint (which was not certified by peer review) is the author/funder, who has granted medRxiv a license to display the preprint in perpetuity.

It is made available under a CC-BY-NC-ND 4.0 International license .

6. Kallet RH. A Comprehensive Review of Prone Position in ARDS. Respir Care. 2015;60:1660-1687.

7. Lee DL, Chiang HT, Lin SL, Ger LP, Kun MH, Huang YC. Proneposition ventilation induces sustained improvement in oxygenation in patients with acute respiratory distress syndrome who have a large shunt. Crit Care Med 2002;30:1446-1452. 
Plateau Airway Pressure
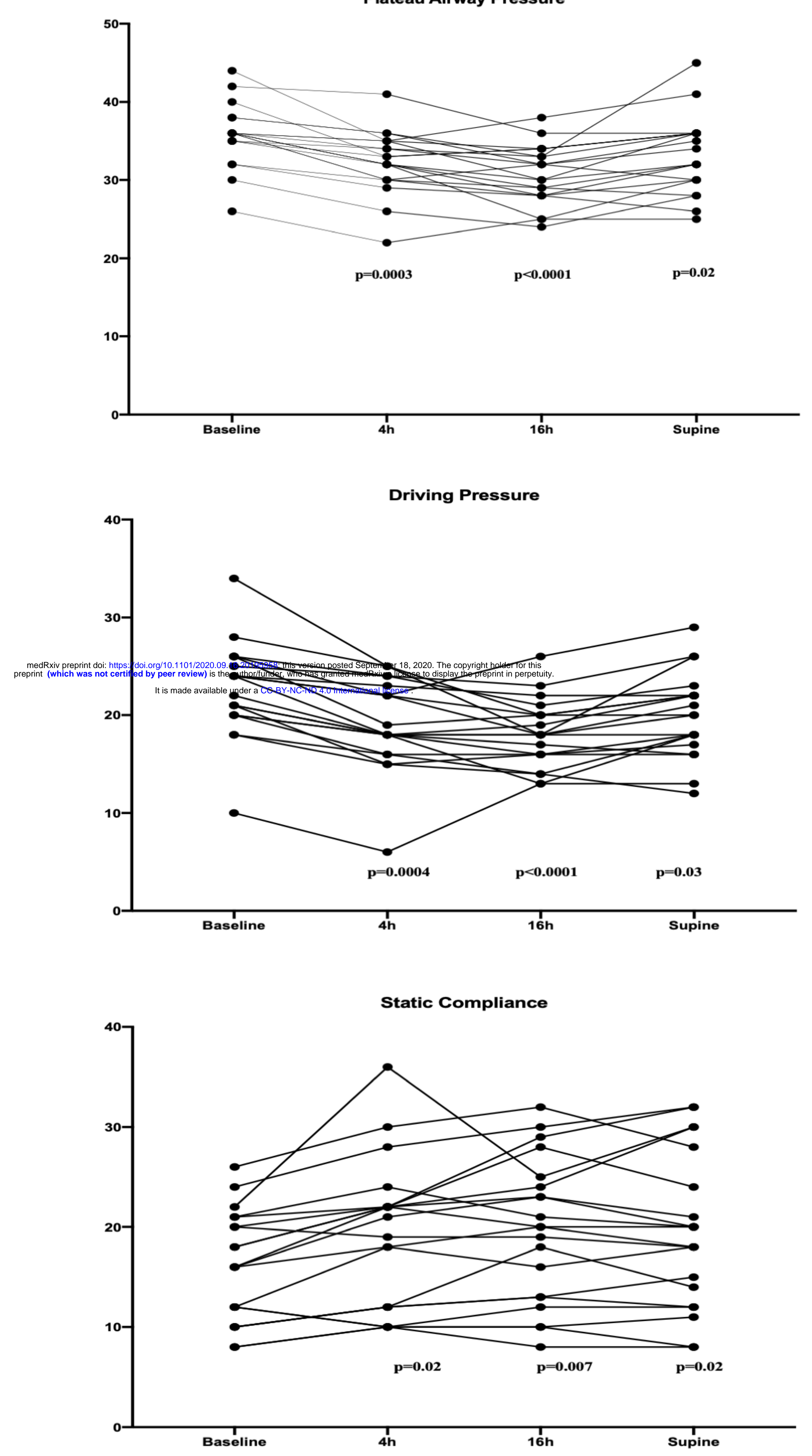
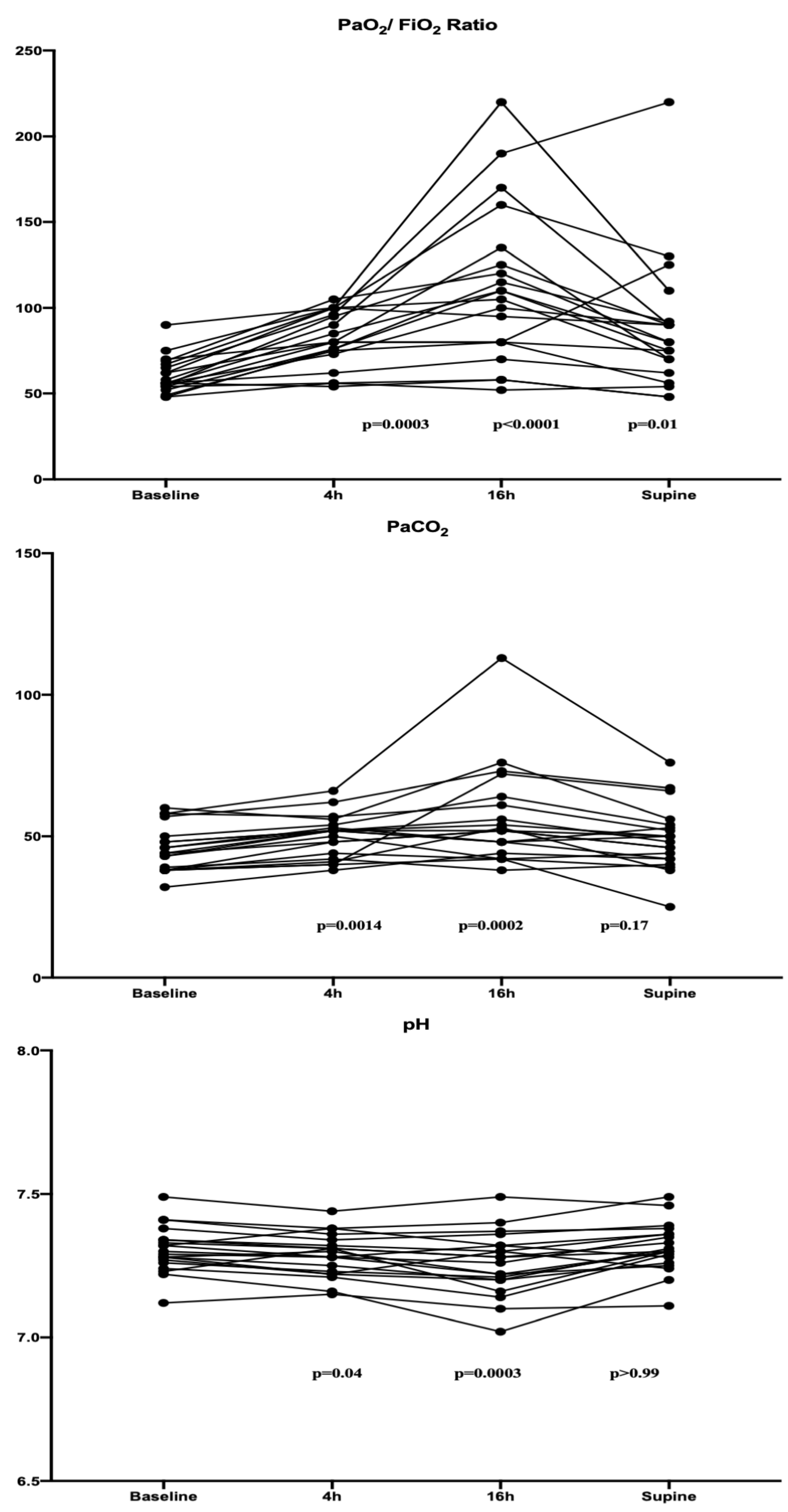